International Electronic Journal of Algebra

VOLUME 21 (2017) 39-54

\title{
PROPERTIES OF RING EXTENSIONS INVARIANT UNDER GROUP ACTION
}

\author{
Amy Schmidt \\ Received: 18 January 2016 \\ Communicated by A. Çiğdem Özcan
}

\begin{abstract}
Let $G$ be a subgroup of the automorphism group of a commutative ring with identity $T$. Let $R$ be a subring of $T$. We show that $R^{G} \subset T^{G}$ is a minimal ring extension whenever $R \subset T$ is a minimal extension under various assumptions. Of the two types of minimal ring extensions, integral and integrally closed, both of these properties are passed from $R \subset T$ to $R^{G} \subseteq T^{G}$. An integrally closed minimal ring extension is a flat epimorphic extension as well as a normal pair. We show that each of these properties also pass from $R \subset T$ to $R^{G} \subseteq T^{G}$ under certain group action.
\end{abstract}

Mathematics Subject Classification (2010): 13A50, 13B02

Keywords: Fixed ring, ring of invariants, invariant theory, locally finite, minimal ring extension, flat epimorphism, normal pair

\section{Introduction}

All rings herein are commutative with identity, and all homomorphisms and subrings are unital. For a ring $R$, we denote by $\operatorname{Reg}(R)$ the set of regular elements; $\operatorname{Spec}(R)$ the set of prime ideals; $\operatorname{Max}(R)$ the set of maximal ideals; $\operatorname{Rad}_{R}(I)$ the radical in $R$ of an ideal $I \subset R$; $\operatorname{tq}(R)$ the total quotient ring; $\mathrm{qf}(R)$ the quotient field, if $R$ is a domain; and $\operatorname{Aut}(R)$ the automorphism group of $R$. As in [11], we refer to the lying-over, going-up, and incomparable properties of ring extensions as LO, GU, and INC, respectively.

Given a subgroup $G$ of $\operatorname{Aut}(R)$, we say that $G$ acts on $R$ and denote the fixed ring of this action by $R^{G}=\{r \in R \mid \sigma(r)=r$ for all $\sigma \in G\}$. We say that a property of $R$ is $\left(G\right.$-)invariant if $R^{G}$ also has the property. Our purpose in this paper is to enhance the popular investigation of which ring-theoretic properties are invariant. As the title of this paper suggests, we determine properties of the ring extension $R \subset T$ that are $(G)$-invariant when we consider $G$ is a subgroup of $\operatorname{Aut}(T)$ (not necessarily a subgroup of $\operatorname{Aut}(R)$ ), and $R^{G}:=R \cap T^{G}$. (In fact this definition agrees with our original definition of $R^{G}$ even though $G$ is not assumed 
to be a subgroup of $\operatorname{Aut}(R)$.) We say that a property of the extension $R \subset T$ is invariant if $R^{G} \subseteq T^{G}$ also has the property.

Our riding assumptions in this work are that $R$ is a subring of $T$, and that $G$ acts on $T$ via automorphisms. Additionally in Section 2, we assume that $R^{G} \neq T^{G}$, and, in Section 3, we assume that $R$ is $G$-invariant, i.e., $\sigma(R) \subseteq R$ for all $\sigma \in G$. It then follows that $G$ is a subgroup of $\operatorname{Aut}(R)$.

We denote the orbit of $t \in T$ under $G$ by $\mathcal{O}_{t}$, i.e., $\mathcal{O}_{t}=\{\sigma(t) \mid \sigma \in G\}$, and we define

$$
n_{t}:=\left|\mathcal{O}_{t}\right|, \quad \hat{t}:=\sum_{t_{i} \in \mathcal{O}_{t}} t_{i} \quad \text { and } \quad \tilde{t}:=\prod_{t_{i} \in \mathcal{O}_{t}} t_{i} .
$$

If $G$ is finite, instead we denote by $\hat{t}$ the sum $\sum_{\sigma \in G} \sigma(t)$ (allowing for duplicates). We say that $G$ is locally finite (on $T$ ) if $\mathcal{O}_{t}$ is finite for all $t \in T$. Note that if $G$ is locally finite on $T$, it is not necessarily locally finite on $R$ unless $R$ is $G$-invariant.

Given an ideal $I \subset T$ we denote the orbit of $I$ under $G$ by $\mathcal{O}_{I}:=\{\sigma(I) \mid \sigma \in G\}$. By the First Isomorphism Theorem, $T / I \cong T / \sigma(I)$. Clearly, $T / I$ is a field (domain) if and only if $T / \sigma(I)$ is a field (domain). Hence, $I$ is a maximal (prime) ideal if and only if $\sigma(I)$ is a maximal (prime) ideal. We say that $G$ is strongly locally finite (on $T$ ) if $G$ is locally finite and $\mathcal{O}_{P}$ is finite for all $P \in \operatorname{Spec}(T)$. If $G$ is strongly locally finite on $T$, it is not necessarily strongly locally finite on $R$ even if $R$ is $G$-invariant. To see this define $R$ and $G$ as in [7, Example 2.3], and set $T:=\mathrm{qf}(R)$.

As in [9], we say that $R \subset T$ is a minimal ring extension if there is no ring $S$ such that $R \subset S \subset T$. Clearly, this is true if and only if $T=R[u]$ for all $u \in T \backslash R$. Since $R \subseteq \bar{R} \subseteq T$, where $\bar{R}$ is the integral closure of $R$ in $T$, if $R \subset T$ is minimal, then either $R$ is integrally closed in $T$, or $T$ is integral over $R$ (equivalently, $T$ is module finite over $R$ ). In the first case we call $R \subset T$ an integrally closed minimal ring extension, and in the second case, we call it an integral minimal ring extension. By [9, Théorème 2.2], if $R \subset T$ is a minimal ring extension, there exists a unique maximal ideal $M$ of $R$ such that $R_{P} \cong T_{P}$ for all $P \in \operatorname{Spec}(T) \backslash\{M\}$. This maximal ideal is commonly referred to as the crucial maximal ideal of the extension.

In [14] (cf. [5]), Picavet and Picavet-L'Hermitte classify integral minimal ring extensions in terms of the crucial maximal ideal. In [2], Cahen et al. characterize integrally closed minimal ring extensions in terms of a certain "critical ideal." We utilize these results and provide major theorems and definitions for reference in Sections 2 and 3 .

In Section 2, under the assumptions that $R \subset T$ is an integral minimal ring extension and that $G$ is locally finite acting on $T$, we show that $R^{G} \subset T^{G}$ is an integral minimal ring extension under mild hypotheses. To do so we use $[14$, 
Theorem 3.3], given in Theorem 2.6 for reference. We present examples to show that it is necessary to assume that $R^{G} \neq T^{G}$. In one example, we use the idealization construction. Given a ring $R$ and an $R$-module $M$, the idealization $R(+) M=$ $\{(r, m) \mid r \in R, m \in M\}$ is a ring with multiplication given by $(r, m)\left(r^{\prime}, m^{\prime}\right)=$ $\left(r r^{\prime}, r m^{\prime}+r^{\prime} m\right)$ and componentwise addition. By [4, Theorem 2.4], $R(+) M$ is a minimal ring extension of $R$ if and only if $M$ is a simple $R$-module.

In Section 3, we turn to the integrally closed case. In Theorem 3.6, we show that the minimal property of an integrally closed ring extension is $G$-invariant assuming that $G$ is locally finite (on $T$, hence on $R$, since $R$ is assumed to be $G$-invariant).

In Section 4, we show that certain properties of ring extensions related to minimality are also invariant. We consider integral extensions, integrally closed extensions, flat epimorphic extensions, and normal pairs.

\section{Integral minimal ring extensions}

We begin with a well-known result that is fundamental in this work and in much of the work by Dobbs and Shapiro [6], [7], [8]. These papers on invariant theory are a strong influence on our work.

Lemma 2.1. If $G$ is locally finite (on $T$ ), then $T$ is integral over $T^{G}$.

Recall that our riding assumptions in this section are that $R \subset T, G \leq \operatorname{Aut}(T)$, and $R^{G} \neq T^{G}$. In the following lemma we establish several technical results needed for the main result of this section. Proposition 2.3 is another tool for the main result and is also of independent interest.

Lemma 2.2. Assume that $G$ is locally finite (on $T$ ) and that $M:=\left(R:_{R} T\right.$ ) is a maximal ideal of $R$. Set $\mathrm{M}:=M \cap R^{G}=M \cap T^{G}$.

(a) If $R$ is integral over $R^{G}$, then the conductor $\left(R^{G}:_{R^{G}} T^{G}\right)$ equals $\mathrm{M}$.

(b) If there exist $N \in \operatorname{Spec}(T)$ containing $M$, then $M=N \cap R$.

Proof. (a) Let $x \in \mathrm{M}$. Then $x \in R^{G}$, and $x t \in R$, for all $t \in T$. If $t \in T^{G}$, then $x t \in T^{G}$, from which it follows that $x t \in T^{G} \cap R=R^{G}$. Hence $x \in\left(R^{G}:_{R^{G}} T^{G}\right)$. Thus $\mathrm{M} \subseteq\left(R^{G}:_{R^{G}} T^{G}\right)$. Since $R$ is integral over $R^{G}$, we have that $\mathrm{M} \in \operatorname{Max}\left(R^{G}\right)$. Thus $\mathrm{M}=\left(R^{G}:_{R^{G}} T^{G}\right)$.

(b) Clearly $M=N \cap R$ whenever $N$ is a prime ideal of $T$ containing $M$, since $M \in \operatorname{Max}(R)$.

Proposition 2.3. Let $M \in \operatorname{Max}(R)$ and $\mathrm{M}:=M \cap R^{G}$. Assume that $G$ is locally finite (on $T$ ) such that $\operatorname{char}\left(R^{G} / \mathrm{M}\right) \nmid n_{r}$ for all $r \in R$. If the orbit of $M$ in 
$R$ is a singleton set, i.e., $\mathcal{O}_{M}=\{M\}$, then the $G$-action extends to $R / M$ via $\sigma(r+M)=\sigma(r)+M$, for $\sigma \in G$. Moreover, if $R$ is integral over $R^{G}$, then $R^{G} / \mathrm{M} \cong(R / M)^{G}$.

Proof. The given action of $G$ on $R / M$ is well-defined; if $r+M=s+M$, then $\sigma(r)-\sigma(s) \in \sigma(M)=M$. Hence $\sigma(r)+M=\sigma(s)+M$.

As for the moreover, first note that $\mathrm{M} \in \operatorname{Max}\left(R^{G}\right)$ by integrality. Define $\phi$ : $R^{G} / \mathrm{M} \rightarrow(R / M)^{G}$ by $r+\mathrm{M} \mapsto r+M$. Clearly $\phi$ is a ring homomorphism. If $\phi(r+\mathrm{M})=0+M$, then $r \in M$. It follows that $r \in M \cap R^{G}=\mathrm{M}$, so $r+\mathrm{M}=0+\mathrm{M}$. Hence $\phi$ is injective.

Now let $r+M \in(R / M)^{G}$. Then $r+M=\sigma(r)+M$ for all $\sigma \in G$. Summing the elements of $\mathcal{O}_{r}$ we have that $n_{r} r+M=\hat{r}+M$. Since $R / M$ is a field, we have that $r+M=\left(n_{r}+M\right)^{-1}(\hat{r}+M)$. Similarly, since $n_{r}+\mathrm{M} \in R^{G} / \mathrm{M}$, we have that $y+\mathrm{M}:=\left(n_{r}+\mathrm{M}\right)^{-1} \in R^{G} / \mathrm{M}$. It follows that $y+M=\left(n_{r}+M\right)^{-1}$, whence $\phi(y \hat{r}+\mathrm{M})=y \hat{r}+M=\left(n_{r}+M\right)^{-1}(\hat{r}+M)=r+M$. Thus $\phi$ is surjective. Hence $R^{G} / m \cong(R / M)^{G}$.

The technique of averaging the orbit of an element used above to produce $r+M=$ $\left(n_{r}+M\right)^{-1}(\hat{r}+M)$ is introduced in [1]. We generalize this method in the following lemma.

Lemma 2.4. Assume that $G$ is locally finite (on $T$ ). Let $t \in T^{G}$. We show that if $0 \neq t=r_{1} u_{1}+r_{2} u_{2}+\cdots+r_{k} u_{k}$ for some $r_{i} \in R$ and $u_{i} \in T^{G}$, then there exist $m, m_{i} \in \mathbb{N}$ and $r_{i}^{\prime} \in R^{G}$ such that $0 \neq m t=m_{1} r_{1}^{\prime} u_{1}+m_{2} r_{2}^{\prime} u_{2}+\cdots+m_{k} r_{k}^{\prime} u_{k}$ whenever

(a) $T$ is a domain and $\operatorname{char}(T)=0$ or $\operatorname{char}(T) \nmid n_{t}$ for all $t \in T$, or

(b) $|G|$ is finite and a unit in $T$.

Proof. For all $t \in T$, fix a subset $\mathcal{N}_{t}$ of $G$ such that for each $a \in \mathcal{O}_{t}$ there exists a unique $\sigma \in \mathcal{N}_{t}$ with $a=\sigma(t)$ (and so $\left|\mathcal{N}_{t}\right|=\left|\mathcal{O}_{t}\right|=n_{t}$ ).

First we show that if

$$
0 \neq t=q_{1} u_{1} \cdots+q_{i} u_{i}+r_{i+1} u_{i+1}+\cdots+r_{k} u_{k},
$$

where $t \in T^{G}, q_{i} \in R^{G}$, and $r_{j} \in R$, then there exists $m \in \mathbb{N}, r_{i+1}^{\prime} \in R^{G}$, and $s_{j} \in R$ such that

$$
0 \neq m t=m\left(q_{1} u_{+} \cdots+q_{i} u_{i}\right)+r_{i+1}^{\prime} u_{i+1}+s_{i+2} u_{i+2}+\cdots+s_{k} u_{k} .
$$


Applying each $\sigma \in \mathcal{N}_{r_{i+1}}$ to (1) and summing establishes (2). In particular,

$$
m=n_{r_{i+1}}, \quad r_{i+1}^{\prime}=\widehat{r_{i+1}}, \quad \text { and } \quad s_{j}=\sum_{\sigma \in \mathcal{N}_{r_{i+1}}} \sigma\left(r_{j}\right) u_{j},
$$

for $i+2 \leq j \leq k$. Note that $n_{r_{i+1}} t \neq 0$ under assumption (a). Since $i=1$ establishes the base case, the assertion of the lemma now follows from induction. Under assumption (b), the same argument holds replacing $\mathcal{N}_{r_{i+1}}$ with $G$ and $n_{r_{i+1}}$ with $|G|$.

Remark 2.5. The result in case (a) above also holds if $(T, M)$ is a quasilocal ring and characteristic is defined as $p \in \mathbb{Z}$ such that $M \cap \mathbb{Z}=p \mathbb{Z}$. Moreover, the assumption that $\operatorname{char}(T)=0$ or $\operatorname{char}(T) \nmid n_{t}$ for all $t \in T$ is more common than it may appear. In fact, condition (b) implies (a), and, in related work on rings of invariants, condition (b) is a conventional assumption.

We have established the machinery needed to prove the main result of this section. We use the characterization provided below for reference.

Theorem 2.6. [14, Theorem 3.3] (cf. [5, Corollary II.2]) Let $R \rightarrow T$ be an injective ring homomorphism, with conductor $\left(R:_{R} T\right)$. Then $R \rightarrow T$ is minimal and finite if and only if $\left(R:_{R} T\right) \in \operatorname{Max}(R)$ and one of the following three conditions holds:

(a) Inert case: $\left(R:_{R} T\right) \in \operatorname{Max}(T)$ and $R /\left(R:_{R} T\right) \rightarrow T /\left(R:_{R} T\right)$ is a minimal field extension.

(b) Decomposed case: There exist $N_{1}, N_{2} \in \operatorname{Max}(T)$ such that $\left(R:_{R} T\right)=$ $N_{1} \cap N_{2}$ and the natural maps $R /\left(R:_{R} T\right) \rightarrow T / N_{1}$ and $R /\left(R:_{R} T\right) \rightarrow T / N_{2}$ are each isomorphisms.

(c) Ramified case: There exists $N \in \operatorname{Max}(T)$ such that $N^{2} \subseteq\left(R:_{R} T\right) \subset N$, $\left[T /\left(R:_{R} T\right): R /\left(R:_{R} T\right)\right]=2$ and the natural map $R /\left(R:_{R} T\right) \rightarrow T / N$ is an isomorphism.

We now present our main result on the invariance of minimality of integral ring extensions.

Theorem 2.7. Let $R \subset T$ be an integral minimal extension with crucial maximal ideal $M=\left(R:_{R} T\right)$. Assume that $G$ is locally finite (on $\left.T\right)$ such that $\operatorname{char}\left(R^{G} /(M \cap\right.$ $\left.\left.T^{G}\right)\right) \nmid n_{r}$, for all $r \in R$. Also assume that $R$ is integral over $R^{G}$ and $\mathcal{O}_{M}=\{M\}$ (e.g., if $R$ is $G$-invariant). Then $R^{G} \subset T^{G}$ is a minimal extension of the same type as $R \subset T$. Moreover, the crucial maximal ideal of $R^{G} \subset T^{G}$ is $\left(R^{G}:_{R^{G}} T^{G}\right)$.

Proof. Throughout the argument, set $\mathrm{M}:=\left(R^{G}: R^{G} T^{G}\right)$, whence $\mathrm{M}=M \cap R^{G}=$ $M \cap T^{G}$, by Lemma $2.2(\mathrm{a})$. 
Inert case: By Theorem 2.6(a), $M \in \operatorname{Max}(T)$ and $R / M \rightarrow T / M$ is a minimal field extension. By Proposition 2.3, we may pass to $R / M \subset T / M$ (since $\mathcal{O}_{M}=$ $\{M\})$. Replacing $R / M \subset T / M$ with $R \subset T$, we show that $T^{G}=R^{G}[u]$ for all $u \in T^{G} \backslash R^{G}$, i.e., $R^{G} \subset T^{G}$ is a minimal field extension. If $u \in T^{G} \backslash R^{G}$, then $u \in T \backslash R$, so $T=R[u]$. Let $0 \neq t \in T^{G}$. Then $t=r_{k} u^{k}+\cdots+r_{1} u+r_{0}$, for some $k \in \mathbb{N}$ and $r_{i} \in R$. By Lemma 2.4, there exist $m, m_{i} \in \mathbb{N}$ and $r_{i}^{\prime} \in R^{G}$ such that $0 \neq m t=m_{k} r_{k}^{\prime} u^{k}+\cdots+m_{1} r_{1}^{\prime} u+m_{0} r_{0}^{\prime}$. Since $R^{G}$ is a field, we have that $t=m^{-1}\left(m_{k} r_{k}^{\prime} u^{k}+\cdots+m_{1} r_{1}^{\prime} u+m_{0} r_{0}^{\prime}\right) \in R^{G}[u]$. Hence, $R^{G} \subset T^{G}$ is a minimal field extension. By Theorem 2.6(a), the original fixed ring extension (before passing to the quotient ring extension) $R^{G} \subset T^{G}$ is an inert integral minimal extension with crucial maximal ideal $\mathrm{M}=\left(R^{G}:_{R^{G}} T^{G}\right)$.

Decomposed case: By Theorem 2.6(b), there exist $N_{1}, N_{2} \in \operatorname{Max}(T)$ such that $M=N_{1} \cap N_{2}$ and the natural maps $R / M \rightarrow T / N_{1}$ and $R / M \rightarrow T / N_{2}$ are isomorphisms. Set $\mathrm{N}_{1}:=N_{1} \cap T^{G}$ and $\mathrm{N}_{2}:=N_{2} \cap T^{G}$. By Lemma 2.1, $T$ is integral over $T^{G}$, whence $\mathrm{N}_{1}, \mathrm{~N}_{2} \in \operatorname{Max}\left(T^{G}\right)$. Clearly

$$
\mathrm{M}=M \cap T^{G}=\left(N_{1} \cap N_{2}\right) \cap T^{G}=\mathrm{N}_{1} \cap \mathrm{N}_{2} .
$$

Define $\phi: R^{G} / \mathrm{M} \rightarrow T^{G} / \mathrm{N}_{1}$ via the natural map $r+\mathrm{M} \mapsto r+\mathrm{N}_{1}$. Suppose that $\phi(r+\mathrm{M})=0+\mathrm{N}_{1}$ for some $r \in R^{G}$. Then $r \in \mathrm{N}_{1} \cap R^{G}$, but, by Lemma 2.2(b), $\mathrm{N}_{1} \cap R^{G}=\mathrm{M}$. Hence $r+\mathrm{M}=0+\mathrm{M}$. Thus $\phi$ is injective.

To show that $\phi$ is surjective, we first note that the $G$-action extends to $T / N_{1}$, since it extends to $R / M$ and $R / M \cong T / N_{1}$. From Proposition 2.3, we have that $R^{G} / \mathrm{M} \cong(R / M)^{G} \cong\left(T / N_{1}\right)^{G}$. Let $t+\mathrm{N}_{1} \in T^{G} / \mathrm{N}_{1}$ be nonzero. Then $t+N_{1} \in$ $\left(T / N_{1}\right)^{G}$ is nonzero. (Clearly it is fixed, and if $t \in N_{1}$, then $t \in N_{1} \cap T^{G}=\mathrm{N}_{1}$ - contradiction.) Since $R^{G} / m \cong\left(T / N_{1}\right)^{G}$ (via composition of the natural maps), there exists $r+\mathrm{M} \in R^{G} / \mathrm{M}$ such that $r+\mathrm{M} \mapsto r+M \mapsto r+N_{1}=t+N_{1}$. It follows that $(r-t) \in N_{1} \cap T^{G}=\mathrm{N}_{1}$. Hence $\phi(r+\mathrm{M})=r+\mathrm{N}_{1}=t+\mathrm{N}_{1}$, so $\phi$ is surjective. Thus $R^{G} / \mathrm{M} \cong T^{G} / \mathrm{N}_{1}$. The same argument applies to show $R^{G} / \mathrm{M} \cong T^{G} / \mathrm{N}_{2}$. By Theorem 2.6(b), $R^{G} \subset T^{G}$ is a decomposed integral minimal extension with crucial maximal ideal $\mathrm{M}=\left(R^{G}:_{R^{G}} T^{G}\right)$.

Ramified case: By Theorem 2.6(c), there exists $N \in \operatorname{Max}(T)$ such that $N^{2} \subseteq$ $M \subset N,[T / M: R / M]=2$ and the natural map $R / M \rightarrow T / N$ is an isomorphism. Set $\mathrm{N}:=N \cap T^{G}$, and recall $\mathrm{M}=M \cap T^{G}$. Clearly, $\mathrm{N} \in \operatorname{Max}\left(T^{G}\right)$ and $\mathrm{M} \subsetneq \mathrm{N}$, since $\mathrm{M} \notin \operatorname{Max}\left(T^{G}\right)$ (since $M \notin \operatorname{Max}(T), N \in \operatorname{Max}(T)$, and $T$ is integral over $\left.T^{G}\right)$. For the other containment, let $x \in \mathrm{N}^{2}$. Then $x \in N^{2}$, so $x \in M$. Hence $x \in M \cap T^{G}=\mathrm{M}$. Thus $\mathrm{N}^{2} \subseteq \mathrm{M}$. 
We show that the natural map $\phi: R^{G} / \mathrm{M} \rightarrow T^{G} / \mathrm{N}$ given by $r+\mathrm{M} \mapsto r+\mathrm{N}$ is an isomorphism. Suppose that $\phi(r+\mathrm{M})=0+\mathrm{N}$ for some $r \in R^{G}$. Then $r \in \mathrm{N}$, so $r^{2} \in \mathrm{N}^{2}$. Since $\mathrm{N}^{2} \subseteq \mathrm{M}$ and $\mathrm{M}$ is prime (maximal) in $R^{G}$, we have that $r \in \mathrm{M}$. (Alternatively, $r \in \mathrm{N} \cap R^{G}=\mathrm{M}$, by Lemma 2.2(b).) Hence $r+\mathrm{M}=0+\mathrm{M}$. Thus $\phi$ is injective.

Next we show that $\phi$ is surjective. Let $t+\mathrm{N} \in T^{G} / \mathrm{N}$. Then $t+N \in(T / N)^{G}$. Note that, as in the decomposed case, since $R / M \cong T / N$, the $G$-action extends to $T / N$. From this and from Proposition 2.3, it follows that $R^{G} / \mathrm{M} \cong(R / M)^{G} \cong(T / N)^{G}$ via $r+\mathrm{M} \mapsto r+M \mapsto r+N$. Hence, there exists $r+\mathrm{M} \in R^{G} / \mathrm{M}$ such that $r+\mathrm{M} \mapsto r+M \mapsto r+N=t+N$, from which it follows that $(r-t) \in N \cap T^{G}=\mathrm{N}$. Hence $\phi(r+\mathrm{M})=t+\mathrm{N}$. Thus $\phi$ is surjective.

It remains to show that $\left[T^{G} / \mathrm{M}: R^{G} / \mathrm{M}\right]=2$. Note that $T^{G} / \mathrm{M}$ is not a domain, since $\mathrm{N}^{2} \subseteq \mathrm{M} \subset n$ implies $\mathrm{M}=\mathrm{N}$, if $\mathrm{M}$ is prime. Hence $T^{G} / \mathrm{M} \neq R^{G} / \mathrm{M}$, i.e., $\left[T^{G} / \mathrm{M}: R^{G} / \mathrm{M}\right] \geq 2$.

Suppose that $\left[T^{G} / \mathrm{M}: R^{G} / \mathrm{M}\right]>2$, and let $\left\{e_{1}+\mathrm{M}, e_{2}+\mathrm{M}, e_{3}+\mathrm{M}\right\}$ be an $R^{G} / \mathrm{M}-$ linearly independent set in $T^{G} / \mathrm{M}$. Then each $e_{i} \notin M$; otherwise, $e_{i} \in M \cap T^{G}=\mathrm{M}$. Hence each $e_{i}+M$ is nonzero in $T / M$. Since $[T / M: R / M]=2$, without loss of generality we may assume that there exist $t_{1}+M, t_{2}+M \in T / M$ such that

$$
e_{3}+M=\left(t_{1}+M\right)\left(e_{1}+M\right)+\left(t_{2}+M\right)\left(e_{2}+M\right)=t_{1} e_{1}+t_{2} e_{2}+M .
$$

As in Lemma 2.4, using $\sigma \in \mathcal{N}_{t_{1}}$ and summing $\mathcal{O}_{t_{1}}$ we have that

$$
n_{t_{1}} e_{3}+M=\widehat{t}_{1} e_{1}+\left(\sum_{\sigma \in \mathcal{N}_{t_{1}}} \sigma\left(t_{2}\right)\right) e_{2}+M .
$$

Defining $t_{3}$ to be the coefficient of $e_{2}$ above and repeating the above technique with respect to $t_{3}$ we have that

$$
n_{t_{3}} n_{t_{1}} e_{3}+M=n_{t_{3}} \widehat{t}_{1} e_{1}+\widehat{t}_{3} e_{2}+M \text {. }
$$

It follows that $n_{t_{3}} n_{t_{1}} e_{3}-\left(n_{t_{3}} \widehat{t}_{1} e_{1}+\widehat{t}_{3} e_{2}\right) \in M \cap T^{G}=\mathrm{M}$, so

$$
n_{t_{3}} n_{t_{1}} e_{3}+\mathrm{M}=n_{t_{3}} \widehat{t_{1}} e_{1}+\widehat{t_{3}} e_{2}+\mathrm{M}
$$

Equivalently,

$$
\left(n_{t_{3}} n_{t_{1}}+\mathrm{M}\right)\left(e_{3}+\mathrm{M}\right)=\left(n_{t_{3}} \widehat{t_{1}}+\mathrm{M}\right)\left(e_{1}+\mathrm{M}\right)+\left(\widehat{t_{3}}+\mathrm{M}\right)\left(e_{2}+\mathrm{M}\right)
$$

is an $R^{G} / \mathrm{M}$-linear combination of $e_{1}+\mathrm{M}, e_{2}+\mathrm{M}, e_{3}+\mathrm{M}$ in $T^{G} / \mathrm{M}-$ contradiction. Hence, there cannot exist in $T^{G} / \mathrm{M}$ any more than two $R^{G} / \mathrm{M}$-linearly independent 
elements. Thus $\left[T^{G} / \mathrm{M}: R^{G} / \mathrm{M}\right] \leq 2$. Hence $\left[T^{G} / \mathrm{M}: R^{G} / \mathrm{M}\right]=2$. By Theorem 2.6(c), $R^{G} \subset T^{G}$ is a ramified integral minimal extension with crucial maximal ideal $\mathrm{M}=\left(R^{G}:_{R^{G}} T^{G}\right)$.

Remark 2.8. If we were to assume that $R$ is $G$-invariant in this section (instead of waiting until Section 3), then the conditions that $R$ is integral over $R^{G}$ and $\mathcal{O}_{M}=\{M\}$ would be satisfied. Integrality follows from Lemma 2.1. To see that $\mathcal{O}_{M}=\{M\}$, note that $\sigma(M) T=\sigma(M T) \subseteq \sigma(R)=R$, for any $\sigma \in G$. Hence $\sigma(M) \subseteq M$. Since $\sigma(M) \in \operatorname{Max}(R)$, we have that $\sigma(M)=M$.

Remark 2.9. It is necessary to assume that $R^{G} \neq T^{G}$ in this section, as illustrated in the following example.

Example 2.10. The fixed rings are equal, even under finite group action, in the following cases:

Inert case: Set $R:=\mathbb{R}, T:=\mathbb{C}$, and $G:=\{1, \sigma\}$, where $\sigma$ is the conjugacy map. Then $R^{G}=R=T^{G}$.

Decomposed case: Let $F$ be a field such that $\operatorname{char}(F) \neq 2$, and set $R:=$ $\{(x, x) \mid x \in F\}$ and $T:=F \times F . B y[9$, Lemme 1.2(b)], $R \subset T$ is a minimal extension. Define $G:=\{1, \sigma\}$, where $\sigma\left((x, x)=(x,-x)\right.$. Then $R^{G}=T^{G}$.

Ramified case: Let $F$ and $R$ be as above, and set $T:=F(+) F$. Then by $[9$, Lemme 1.2(c)], $R \subset T$ is a minimal extension. Define $G$ as above. Then $R^{G}=T^{G}$.

\section{Integrally closed minimal extension}

Our riding assumptions in this section are that $R$ is a subring of $T$, $G$ acts on $T$ via automorphisms, and $R$ is $G$-invariant. Note that since $R$ is $G$-invariant, if $G$ is locally finite on $T$, then it is also locally finite on $R$. Therefore, throughout this section we refer to "locally finite" as being locally finite on both rings. We show that minimality of an integrally closed extension $R \subset T$ is invariant under locally finite $G$-action. This generalizes Dobbs' and Shapiro's result that the property is invariant if $R$ is a domain and if $|G|$ is finite and a unit in $R$ [7, Theorem 3.6].

Whereas crucial maximal ideals are historically essential to the study of minimal extensions, Cahen et al. introduce critical ideals and use them extensively in characterizing integrally closed minimal extensions of an arbitrary ring [2]. They define a critical ideal for $R \subset T$ as an ideal $I \subset R$ such that $I=\operatorname{Rad}_{R}\left(\left(R:_{R} t\right)\right)$ for all $t \in T \backslash R$. That is, $\operatorname{Rad}_{R}\left(\left(R:_{R} t\right)\right)$ is the same ideal for all $t \in T \backslash R$. They show in [2, Lemma 2.11] that if an extension has a critical ideal, then the ideal is 
prime. Moreover, they show that if $R \subset T$ is a minimal extension, then the critical ideal exists [2, Proposition 2.14(2)] and is maximal [2, Theorem 3.5]. If $R \subset T$ has a critical ideal, we show that $R^{G} \subset T^{G}$ has a critical ideal under any $G$-action such that $R^{G} \neq T^{G}$.

Lemma 3.1. Let $P$ be the critical ideal of $R \subset T$. If $R^{G} \neq T^{G}$, then $\mathrm{P}:=P \cap R^{G}$ is the critical ideal of $R^{G} \subset T^{G}$.

Proof. Let $t \in T^{G} \backslash R^{G}$. Then $t \in T \backslash R$. Hence $P=\operatorname{Rad}_{R}\left(\left(R:_{R} t\right)\right)$, from which it follows that

$$
\mathrm{P}=\operatorname{Rad}_{R}\left(\left(R:_{R} t\right)\right) \cap R^{G}=\operatorname{Rad}_{R^{G}}\left(\left(R:_{R} t\right) \cap R^{G}\right)=\operatorname{Rad}_{R^{G}}\left(\left(R^{G}:_{R^{G}} t\right)\right) .
$$

Thus P is the critical ideal of $R^{G} \subset T^{G}$.

We next show that if a critical ideal is maximal, then its orbit (under $G$ ) is a singleton set.

Lemma 3.2. Suppose that $M$ is the critical ideal for $R \subset T$. If $M$ is a maximal ideal of $R$, then $\sigma(M)=M$ for all $\sigma \in G$, i.e. $\mathcal{O}_{M}=\{M\}$.

Proof. Let $\sigma \in G$ and $t \in T \backslash R$. Note that $\sigma^{-1}(t) \in T \backslash R$; otherwise, if $\sigma^{-1}(t) \in R$, then $t=\sigma\left(\sigma^{-1}(t)\right) \in \sigma(R)=R$ - contradiction. Since $M$ is the critical ideal for $R \subset T, M=\operatorname{Rad}_{R}\left(\left(R:_{R} \sigma^{-1}(t)\right)\right)$. Let $r$ be an arbitrary element of $R$, let $x \in M$, and set $y:=\sigma^{-1}(x)$. Then there exists $n \in \mathbb{N}$ such that $x^{n} r \in R$, from which it follows that $\left(\sigma^{-1}(x)\right)^{n} \sigma^{-1}(t) \in \sigma^{-1}(R)=R$. Hence $y=\sigma^{-1}(x) \in \operatorname{Rad}_{R}\left(\left(R:_{R}\right.\right.$ $\left.\left.\sigma^{-1}(t)\right)\right)=M$. Thus $x=\sigma(y) \in \sigma(M)$, which shows that $M \subseteq \sigma(M)$. Since $M$ is maximal, $M=\sigma(M)$, as desired.

Remark 3.3. It is not necessary to assume that $M$ is maximal in the preceding lemma. A similar set-theoretic argument establishes the converse $\sigma(M) \subseteq M$.

Related to critical ideals are valuation pairs for an extension $R \subset T$. As in [13], for $P \in \operatorname{Spec}(R),(R, P)$ is a valuation pair of $T$ if there is a valuation $v$ on $T$ with $R=\{t \in T \mid v(t) \geq 0\}$ and $P=\{t \in T \mid v(t)>0\}$. Equivalently, $(R, P)$ is a valuation pair of $T$ if $R=S$ whenever $S$ is an intermediate ring containing a prime ideal lying over $P$ [13]. Rank 1 valuation pairs are one of several equivalences of integrally closed minimal extensions in [2]. The rank of a valuation pair $(R, P)$ of $T$ is the rank of the valuation group. The following lemma describes the relationship between critical ideals and valuation pairs. 
Lemma 3.4. [2, Lemma 2.12] Let $(R, P)$ be a valuation pair of $T$. Then $R \subset T$ has a critical ideal if an only if $(R, P)$ has rank 1 . Moreover, under these conditions, $P$ is the critical ideal of $R \subset T$.

Our next result is fundamental to the invariance of minimality of integrally closed extensions established in Theorem 3.6.

Proposition 3.5. Assume that $G$ is locally finite such that $R^{G} \neq T^{G}$. Let $M \in$ $\operatorname{Max}(R)$ and set $\mathrm{M}:=M \cap R^{G}$. If $\mathcal{O}_{M}=\{M\}$, then $\left(R^{G}, \mathrm{M}\right)$ is a valuation pair of $T^{G}$ whenever $(R, M)$ is a valuation pair of $T$.

Proof. Let $A$ be a ring such that $R^{G} \subseteq A \subseteq T^{G}$. Then $R \subseteq A R \subseteq T$. First note that $A R$ is integral over $A$, since $R$ is integral over $R^{G}$, hence over $A$. Let $\mathrm{Q} \in \operatorname{Spec}(A)$ such that $\mathrm{Q} \cap R^{G}=\mathrm{M}$, and let $Q \in \operatorname{Spec}(A R)$ lie over Q. From

$$
\mathrm{M}=\mathrm{Q} \cap R^{G}=(Q \cap A) \cap R^{G}=Q \cap R^{G}=(Q \cap R) \cap R^{G}
$$

it follows that $Q \cap R$ is maximal in $R$, by integrality. We claim $Q \cap R=M$. Suppose not. Then there exists $x \in(Q \cap R) \backslash M$, since $Q \cap R$ and $M$ are incomparable (as maximal ideals). It follows that $\tilde{x} \in Q \cap R^{G}=\mathrm{M}=M \cap R^{G}$. Hence $\sigma(x) \in M$ for some $\sigma \in G$. Since $\mathcal{O}_{M}=\{M\}$, we have that $x \in \sigma^{-1}(M)=M$ - contradiction. Hence $Q \cap R=M$. Since $(R, M)$ is a valuation pair of $T$, we have that $A R=R$, whence $A=R^{G}$. Thus $\left(R^{G}, \mathrm{M}\right)$ is a valuation pair of $T^{G}$.

Of the several integrally closed minimal extension equivalences in $[2$, Theorem 3.5], we use the condition that there exists a maximal ideal $M$ such that $(R, M)$ is a rank 1 valuation pair of $T$ where $R \subset T$. With this equivalence, our main result of this section follows easily from the preceding results.

Theorem 3.6. Assume that $G$ is locally finite. If $R \subset T$ is an integrally closed minimal extension, then $R^{G} \subset T^{G}$ is an integrally closed minimal extension.

Proof. First we show that $R^{G} \neq T^{G}$. Let $t \in T \backslash R$. Then $\tilde{t} \in T^{G}$. Suppose that $\tilde{t} \in R^{G}$. Then $\tilde{t} \in R$. By [9, Proposition 3.1], $\sigma(t) \in R$ for some $\sigma \in G$, whence $t=\sigma^{-1}(\sigma(t)) \in \sigma^{-1}(R)=R$ - contradiction. Hence, $\tilde{t} \in T^{G} \backslash R^{G}$. Thus, $R^{G} \subsetneq T^{G}$.

Let $M$ be the critical ideal for $R \subset T$. By Lemma 3.1, $m:=M \cap R^{G}$ is the critical ideal for $R^{G} \subset T^{G}$. Since $R \subset T$ is a minimal extension, the critical ideal $M$ is maximal. By Lemma 3.2 $\mathcal{O}_{M}=\{M\}$. By Lemma $3.5\left(R^{G}, m\right)$ is a valuation pair of $T^{G}$. Since $m$ is the critical ideal of $R^{G} \subset T^{G}$, this valuation pair has rank 1 by Lemma 3.4. Hence, $R^{G} \subset T^{G}$ is an integrally closed minimal extension by $[2$, Proposition 3.5]. 


\section{Minimal extensions, flat epimorphisms, and normal pairs}

In this section, we generalize the results of Sections 2 and 3. Of course, arbitrary integral or integrally closed extensions are a generalization of minimal integral or integrally closed extensions, respectfully. Integrally closed minimal ring extensions are both flat epimorphic extensions [9, Théorème 2.2] and normal pairs. In the following results we establish the invariance of these related properties under various assumptions.

Proposition 4.1. Assume that $R$ is $G$-invariant and $G$ is locally finite (on $T$ and so on $R$ ). If $R \subset T$ is an integral extension, then $R^{G} \subseteq T^{G}$ is an integral extension.

Proof. This follows from Lemma 2.1 and by transitivity [11, Theorem 40].

Proposition 4.2. If $R$ is integrally closed in $T$, then $R^{G}$ is integrally closed in $T^{G}$.

Proof. Let $u \in T^{G}$ be integral over $R^{G}$. Then $u \in T$ is integral over $R$. Hence $u \in T^{G} \cap R=R^{G}$.

In Theorem 2.7 we require a certain restriction of characteristic. Assuming that $|G|$ is finite and a unit in the base ring, we can remove this restriction. Of course, if $G$ is finite, then it is locally finite. Hence, the following result and corollary re-establish Theorem 3.6.

Proposition 4.3. Let $R \subset T$ be a minimal extension. Assume that $G$ is finite such that $|G|$ is a unit in $R$ and $R^{G} \neq T^{G}$. Then $R^{G} \subset T^{G}$ is a minimal extension.

Proof. Let $u \in T^{G} \backslash R^{G}$. Clearly, $u \in T \backslash R$. Hence, $T=R[u]$. Let $t \in T^{G}$. Then $t=r_{n} u^{n}+\cdots+r_{1} u+r_{0}$ for some $r_{i} \in R$. Applying the averaging technique introduced in Section 2 we have that

$$
t=|G|^{-1} \sum_{\sigma \in G} \sigma\left(r_{n}\right) u^{n}+\cdots+\sigma\left(r_{1}\right) u+\sigma\left(r_{0}\right) .
$$

Thus $T^{G}=R^{G}[u]$, i.e. $R^{G} \subset T^{G}$ is a minimal extension.

Combining Propositions 4.1, 4.2, and 4.3, we have the following corollary.

Corollary 4.4. Under the hypotheses of Proposition 4.3, if $R \subset T$ is an integral or integrally closed minimal extension, then $R^{G} \subset T^{G}$ is an integral or integrally closed minimal extension, respectively. 
Recall a collection of ideals $\mathcal{F}$ of a ring $R$ is a Gabriel filter if it satisfies:

(i) If $I \in \mathcal{F}$ and $I \subseteq J$, then $J \in \mathcal{F}$.

(ii) If $I, J \in \mathcal{F}$, then $I \cap J \in \mathcal{F}$.

(iii) If for an ideal $I$ there exists $J \in \mathcal{F}$ such that $(I: j) \in \mathcal{F}$ for every $j \in J$, then $I \in \mathcal{F}$

For more information on Gabriel filters, see [15]. In particular, see [10] for their use in commutative ring theory. The following theorem illustrates the relationship between these filters and flat epimorphic extensions.

Theorem 4.5. [15, Theorem 2.1, Ch. XI] Let $\phi: R \rightarrow T$ be a ring homomorphism. Then $\phi$ is a flat epimorphism if and only if the collection $\mathcal{F}=\{I \subset R \mid \phi(I) T=T\}$ where $I$ is an ideal in $R$ is a Gabriel filter, and there exists an isomorphism $\psi$ : $T \rightarrow R_{\mathcal{F}}$ such that $\psi \circ \phi: R \rightarrow R_{\mathcal{F}}$ is the canonical homomorphism. Such a filter is called perfect.

By [15, Exercise 8, p. 242], $T$ is a perfect localization of $R$ if and only if for all $t \in T,\left(R:_{R} t\right) T=T$. With this definition and Lemma 4.6 we show that being a perfect localization (equivalently, flat epimorphic extension) is an invariant property in Proposition 4.7.

Lemma 4.6. Assume that $R \subset T, G$ is strongly locally finite (on $T$ ), and $R$ is $G$-invariant. Define $\mathcal{F}:=\{I \subset R \mid I T=T\}$ and $\mathcal{F}^{\prime}:=\left\{J \subset R^{G} \mid J T^{G}=T^{G}\right\}$. If $I \in \mathcal{F}$, then $I \cap R^{G} \in \mathcal{F}^{\prime}$.

Proof. Note that $I \in \mathcal{F}$ if and only if every $P \in \operatorname{Spec}(R)$ containing $I$ is not lain over in $T$. Also note that $\mathcal{F}^{\prime}=\left\{J \subset R^{G} \mid J R \in \mathcal{F}\right\}$. Let $I \in \mathcal{F}$ and let $P \in \operatorname{Spec}(R)$ contain $\left(I \cap R^{G}\right) R$. We claim $I \subseteq \sigma(P)$ for some $\sigma \in G$, whence $P T=$ $\sigma^{-1}(\sigma(P) T)=\sigma^{-1}(\sigma(P T))=T$ (since $\left.I T=T\right)$. Let $x \in I$. Then $\tilde{x} \in I \cap R^{G}$, so $\tilde{x} \in P$. It follows that $\sigma(x) \in P$ for some $\sigma \in G$; equivalently, $x \in \sigma^{-1}(P)$. Hence $I \subseteq \bigcap_{Q \in \mathcal{O}_{P}} Q$. Since $G$ is strongly locally finite, $\mathcal{O}_{P}$ is finite. It follows that $I \subseteq Q$ for some $Q \in \mathcal{O}_{P}$ by the Prime Avoidance Lemma [11, Theorem 81]. Hence the claim is satisfied by $\sigma \in G$, where $Q=\sigma(P)$, so $P T=T$. Thus, every prime containing $\left(I \cap R^{G}\right) R$ is not lain over in $T$. That is, $\left(I \cap R^{G}\right) R \in \mathcal{F}$, whence $I \cap R^{G} \in \mathcal{F}^{\prime}$, as desired.

We are now ready to show that perfect localizations (flat epimorphic extensions) are invariant under strongly locally finite group action using Lemma 4.6.

Theorem 4.7. Assume that $R \subset T, G$ be strongly locally finite (on $T$ ), and $R$ is $G$-invariant. Let $\mathcal{F}$ and $\mathcal{F}^{\prime}$ be as in Lemma 4.6. Then (a) $\mathcal{F}^{\prime}$ is a Gabriel filter 
whenever $\mathcal{F}$ is a Gabriel filter, and (b) if $R \subset T$ is a flat epimorphic extension, then so is $R^{G} \subseteq T^{G}$. In particular, $T^{G}=\left(R^{G}\right)_{\mathcal{F}^{\prime}}$ whenever $T=R_{\mathcal{F}}$.

Proof. (a) Suppose that $\mathcal{F}$ is a Gabriel filter. We check that $\mathcal{F}^{\prime}$ satisfies the defining conditions (i) through (iii) of a Gabriel filter given above. Let $I \in \mathcal{F}^{\prime}$, and let $J$ be an ideal of $R^{G}$ containing $I$. Then $I R \in \mathcal{F}$ and $I R \subseteq J R$, so $J R \in \mathcal{F}$. It follows that $J T=T$, so $J T^{G}=T^{G}$, since $T$ is integral over $T^{G}$. Hence $J \in \mathcal{F}^{\prime}$, which establishes condition (i). Now let $I, J \in \mathcal{F}^{\prime}$. Then $I T=T$ and $J T=T$. Suppose that $I \cap J \notin \mathcal{F}^{\prime}$, i.e. $(I \cap J) T^{G} \neq T^{G}$. Again by integrality, $(I \cap J) T \neq T$. Let $P \in \operatorname{Spec}(T)$ contain $(I \cap J) T$. Then $I \cap J \subseteq P \cap T^{G}=$ : P. It follows that $I \subseteq \mathrm{P}$ or $J \subseteq \mathrm{P}$, but then $I T \subseteq P$ or $J T \subseteq P$ - contradiction. Hence $I \cap J \in \mathcal{F}^{\prime}$, which establishes condition (ii).

It remains to show that $\mathcal{F}^{\prime}$ satisfies condition (iii). Let $J$ be an ideal of $R^{G}$, and suppose that there exists $I \in \mathcal{F}^{\prime}$ such that $\left(J:_{R^{G}} a\right) \in \mathcal{F}^{\prime}$ for all $a \in I$. We claim $\left(J R:_{R} a\right) \in \mathcal{F}$ for all $a \in I R$, whence $J R \in \mathcal{F}$, i.e., $J \in \mathcal{F}^{\prime}$. Let $a:=a_{1} r_{1}+\cdots+a_{n} r_{n} \in I R$, where $a_{i} \in I$ and $r_{i} \in R$. For each $a_{i}$, clearly $\left(J:_{R^{G}}\right.$ $\left.a_{i}\right) R \subseteq\left(\begin{array}{lll}J R:_{R} & a_{i}\end{array}\right)$. Since $\left(J:_{R^{G}} a_{i}\right) \in \mathcal{F}^{\prime}$, we have that $\left(J:_{R^{G}} a_{i}\right) R \in \mathcal{F}$. Hence $\left(J R:_{R} a_{i}\right) \in \mathcal{F}$. From $\left(J R:_{R} a_{i}\right) \subseteq\left(J R:_{R} a_{i} r_{i}\right)$ it follows that $\left(J R:_{R} a_{i} r_{i}\right) \in \mathcal{F}$. Since $\bigcap_{i=1}^{n}\left(J R:_{R} a_{i} r_{i}\right) \in \mathcal{F}$ and $\bigcap_{i=1}^{n}\left(J R:_{R} a_{i} r_{i}\right) \subseteq\left(J R:_{R} a\right)$, we have that $\left(J R:_{R} a\right) \in \mathcal{F}$, proving the claim. Hence $J R \in \mathcal{F}$, i.e. $J \in \mathcal{F}^{\prime}$. Thus $\mathcal{F}^{\prime}$ is a Gabriel filter.

(b) Now we show that $R^{G} \subseteq T^{G}$ is a flat epimorphic extension by showing that $T^{G}$ is a perfect localization of $R^{G}$. Let $x \in T^{G}$. Then $\left(R:_{R} x\right) T=T$, since $T$ is a perfect localization of $R$. It follows that $\left(R:_{R} x\right) \in \mathcal{F}$, and $\left(R:_{R} x\right) \cap R^{G} \in \mathcal{F}^{\prime}$, by Lemma 4.6. We claim $\left(R:_{R} x\right) \cap R^{G} \subseteq\left(R^{G}:_{R^{G}} x\right)$, whence $\left(R^{G}:_{R^{G}} x\right) \in \mathcal{F}^{\prime}$, since $\mathcal{F}^{\prime}$ is a Gabriel filter. Let $y \in(R: R x) \cap R^{G}$. Then $x y \in R$, but $x \in T^{G}$ and $y \in T^{G}$, so $x y \in R^{G}$. Hence $\left(R:_{R} x\right) \cap R^{G} \subseteq\left(R^{G}:_{R^{G}} x\right)$, so $\left(R^{G}:_{R^{G}} x\right) \in \mathcal{F}^{\prime}$ as claimed. (In fact, as the reverse containment clearly holds, $\left(R:_{R} x\right) \cap R^{G}=\left(R^{G}:_{R^{G}} x\right)$.) Thus $\left(R^{G}:_{R^{G}} x\right) T^{G}=T^{G}$, i.e. $T^{G}$ is a perfect localization of $R^{G}$. In particular, $T^{G} \cong\left(R^{G}\right)_{\mathcal{F}}$.

Remark 4.8. (a) The existing literature does not seem to lend itself to utilizing local characterizations of flat epimorphisms in order to establish Theorem 4.7. This can be done under stronger assumptions. Moreover, with such assumptions we can prove the result using only [15, Exercise 8, p. 242].

(b) It would be interesting to know if being an epimorphic extension or a flat extension is an invariant property under any group action. 
Lastly we consider normal pairs which are another generalization of integrally closed minimal extensions. We say that $(R, T)$ is a normal pair if $S$ is integrally closed in $T$ whenever $R \subseteq S \subseteq T$. It would seem as though we could deduce that this is an invariant property of ring extensions from Proposition 4.2 but to do so would require that $A=A R \cap T^{G}$ whenever $R^{G} \subseteq A \subseteq T^{G}$. Instead we use a characterization of normal pairs in terms of extensions satisfying INC.

By [12, Theorem 5.2], $(R, T)$ is a normal pair if and only if $R$ is integrally closed in $T$ and $R \subseteq S$ satisfies INC whenever $R \subseteq S \subseteq T$. We call a pair of rings $(R, T)$ satisfying the latter property an INC-pair and note that it is equivalent to the definition of an INC-pair given in [3].

We have already seen that being an integrally closed extension is an invariant property in Proposition 4.2. To assert that being a normal pair is invariant, it remains to show that being an INC-pair is invariant.

Proposition 4.9. Assume that $G$ is locally finite (on $T$ ) and $R$ is integral over $R^{G}$. If $(R, T)$ is an INC-pair, then $\left(R^{G}, T^{G}\right)$ is an INC-pair.

Proof. Let $R^{G} \subseteq A \subseteq T^{G}$, and let $\mathrm{Q} \subseteq \mathrm{Q}^{\prime}$ be prime ideals of $A$ with the same contraction in $R^{G}$. Set $\mathrm{P}:=\mathrm{Q} \cap R^{G}=\mathrm{Q}^{\prime} \cap R^{G}$. Since $R$ is integral over $R^{G}$ (whence over $A$ ), $A R$ is integral over $A$. Hence, $A \subseteq A R$ satisfies LO and GU. Let $Q \subseteq Q^{\prime}$ be prime ideals in $A R$ such that $\mathrm{Q}=Q \cap A$ and $\mathrm{Q}^{\prime}=Q \cap A$. Setting $P:=Q \cap R$ and $P^{\prime}:=Q^{\prime} \cap R$, we have that $P \subseteq P^{\prime}$ and

$$
P \cap R^{G}=Q \cap R^{G}=(Q \cap A) \cap R^{G}=\mathrm{Q} \cap R^{G}=\mathrm{P},
$$

and $P^{\prime} \cap R^{G}=\mathrm{P}$, by the same reasoning. As an integral extension, $R^{G} \subseteq R$ satisfies INC, whence $P=P^{\prime}$. Since $R \subseteq A R$ satisfies INC, $Q=Q^{\prime}$. Hence $q=q^{\prime}$. Thus $\left(R^{G}, T^{G}\right)$ is an INC-pair.

The corollary below now follows easily from Propositions 4.2 and 4.9.

Corollary 4.10. If $G$ is locally finite (on $T$ ) and $R$ is integral over $R^{G}$, then $\left(R^{G}, T^{G}\right)$ is a normal pair whenever $(R, T)$ is a normal pair.

Remark 4.11. Since normal pairs are Prüfer extensions, and $R \subseteq T$ is called Prüfer if $R \subseteq S$ is a flat epimorphic extension whenever $R \subseteq S \subseteq T$ [12, Theorem 5.2], it may seem as though we could deduce Corollary 4.10 from Theorem 4.7. To do so, however, would require not only that $G$ is strongly locally finite on $T$ but that $S$ is $G$-invariant and $G$ is strongly locally finite on $S$ whenever $R \subseteq S \subseteq T$.

Acknowledgment. The author is immensely grateful to her advisor, Jay Shapiro, for his guidance, suggestions, and help revising the manuscript. 


\section{References}

[1] G. M. Bergman, Hereditary commutative rings and centres of hereditary rings, Proc. London Math. Soc., 23(3) (1971), 214-236.

[2] P.-J. Cahen, D. E. Dobbs and T. G. Lucas, Characterizing minimal ring extensions, Rocky Mountain J. Math., 41(4) (2011), 1081-1125.

[3] D. E. Dobbs, Lying-over pairs of commutative rings, Canad. J. Math., 33(2) (1981), 454-475.

[4] D. E. Dobbs, Every commutative ring has a minimal ring extension, Comm. Algebra, 34(10) (2006), 3875-3881.

[5] D. E. Dobbs, B. Mullins, G. Picavet and M. Picavet-L'Hermitte, On the FIP property for extensions of commutative rings, Comm. Algebra, 33(9) (2005), 3091-3119.

[6] D. E. Dobbs and J. Shapiro, Descent of divisibility properties of integral domains to fixed rings, Houston J. Math., 32(2) (2006), 337-353.

[7] D. E. Dobbs and J. Shapiro, Descent of minimal overrings of integrally closed domains to fixed rings, Houston J. Math., 33(1) (2007), 59-82.

[8] D. E. Dobbs and J. Shapiro, Transfer of Krull dimension, lying-over, and going-down to the fixed ring, Comm. Algebra, 35(4) (2007), 1227-1247.

[9] D. Ferrand and J.-P. Olivier, Homomorphismes minimaux d'anneaux, J. Algebra, 16 (1970), 461-471.

[10] M. Fontana, J. A. Huckaba and I. J. Papick, Prüfer Domains, Monographs and Textbooks in Pure and Applied Mathematics, 203, Marcel Dekker, Inc., New York, 1997.

[11] I. Kaplansky, Commutative Rings, The University of Chicago Press, Chicago, Revised edition, 1974.

[12] M. Knebusch and D. Zhang, Manis Valuations and Prüfer Extensions I, Lecture Notes in Mathematics, 1791, Springer-Verlag, Berlin, 2002.

[13] M. E. Manis, Valuations on a commutative ring, Proc. Amer. Math. Soc., 20 (1969), 193-198.

[14] G. Picavet and M. Picavet-L'Hermitte, Multiplicative Ideal Theory in Commutative Algebra, Chapter About Minimal Morphisms, 369-386, Springer, New York, 2006.

[15] B. Stenström, Rings of Quotients, Springer-Verlag, New York, Heidelberg Berlin, 1975. 


\author{
Amy Schmidt \\ Department of Mathematics \\ Science \& Technology Room 318 \\ Hampton University \\ 100 East Queen Street \\ Hampton, Virginia 23668 \\ e-mail: amy.schmidt@hamptonu.edu
}

